Discussion Paper No. 17-029

Ist eine makroprudenzielle Regulierung des deutschen Hypothekenmarktes geboten?

Oliver Lerbs und Michael Voigtländer

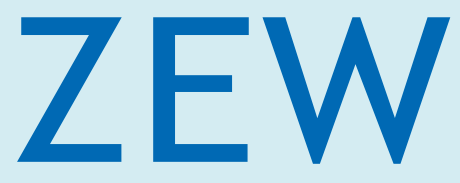

Zentrum für Europäische Wirtschaftsforschung $\mathrm{GmbH}$ Centre for European Economic Research 
Discussion Paper No. 17-029

\title{
Ist eine makroprudenzielle Regulierung des deutschen Hypothekenmarktes geboten?
}

\author{
Oliver Lerbs und Michael Voigtländer
}

Download this ZEW Discussion Paper from our ftp server:

http://ftp.zew.de/pub/zew-docs/dp/dp17029.pdf

Die Discussion Papers dienen einer möglichst schnellen Verbreitung von neueren Forschungsarbeiten des ZEW. Die Beiträge liegen in alleiniger Verantwortung der Autoren und stellen nicht notwendigerweise die Meinung des ZEW dar.

Discussion Papers are intended to make results of ZEW research promptly available to other economists in order to encourage discussion and suggestions for revisions. The authors are solely responsible for the contents which do not necessarily represent the opinion of the ZEW. 


\title{
Ist eine makroprudenzielle Regulierung des deutschen Hypothekenmarktes geboten?
}

\author{
Oliver Lerbs ${ }^{1}$ und Michael Voigtländer ${ }^{2}$
}

\begin{abstract}
Die Bundesregierung hat neue Instrumente der makroprudenziellen Regulierung für den deutschen Hypothekenmarkt geschaffen. Dieser Aufsatz untersucht die Notwendigkeit der Anwendung dieser Instrumente vor dem Hintergrund bestehender Finanzierungsgewohnheiten und Regulierungen. Angesichts der im internationalen Vergleich bereits als konservativ anzusehenden Ausgestaltung der Wohnimmobilienfinanzierung erscheint ein Einsatz makroprudenzieller Instrumente auf absehbare Zeit nicht erforderlich. Grundsätzlich sollten Entscheidungen hierüber regelgebunden auf Grundlage belastbarer Einzelkreditdaten erfolgen. Dafür bedarf es insgesamt eines besseren Monitorings des Immobilienfinanzierungsmarktes.
\end{abstract}

JEL-Klassifikation: E44, E58, G21, G28, R21

Schlüsselwörter: Makroprudenzielle Regulierung, Hypothekenfinanzierung, Hauspreise, Verschuldung, Finanzstabilität

\footnotetext{
${ }^{1}$ Zentrum für Europäische Wirtschaftsforschung (ZEW), Forschungsbereich Internationale Finanzmärkte und Finanzmanagement, Tel. 0621 1235-147, lerbs@zew.de.

2 Institut der deutschen Wirtschaft Köln, Kompetenzfeld Finanzmärkte und Immobilienmärkte, Tel. 0221 4981741, voigtlaender@iwkoeln.de.
} 


\section{Problemstellung und Zielsetzung}

Nach einer langen Phase der Stabilität entwickelt sich der deutsche Wohnimmobilienmarkt ausgesprochen dynamisch. Seit 2010 sind die Kaufpreise für Wohnimmobilien in Deutschland um ca. 30 Prozent angestiegen, in den sieben größten Städten sogar um 60 Prozent (Deutsche Bundesbank, 2017). Das Wachstum der Wohnungsmieten und verfügbaren Einkommen ist dabei deutlich hinter dem Wachstum der Kaufpreise zurück geblieben. Diese Entwicklung kann Auswirkungen auf die Finanzstabilität in Deutschland haben, denn Korrekturen an den Wohnimmobilienmärkten sind in der Wirtschaftsgeschichte immer wieder Auslöser schwerer Finanzkrisen und Rezessionen gewesen. Diese Korrekturen waren besonders folgenschwer, wenn eine Boomphase am Wohnimmobilienmarkt mit einer sprunghaften Ausweitung der Hypothekenkreditvergabe einherging (Reinhart und Rogoff, 2009; Hartmann, 2015). Jüngere Beispiele sind die Immobilienmarktkrisen in mehreren europäischen Ländern ab 2008, die amerikanische Hypothekenkrise ab 2007 sowie die japanische Immobilien- und Aktienkrise ab 1989.

Die Möglichkeiten, eine übermäßige Hypothekenkreditvergabe in Verbindung mit Überbewertungen an Wohnimmobilienmärkten mit klassischen geldpolitischen Instrumenten zu adressieren, sind begrenzt und bergen unerwünschte Nebeneffekte (Kuttner und Shim, 2012; Gelain et al., 2013). Vor diesem Hintergrund setzen Zentralbanken und Aufsichtsbehörden weltweit zunehmend auf makroprudenzielle Regulierungsinstrumente (Crowe et al., 2013; Hiller, 2014). Dabei handelt es sich um aufsichtsrechtliche Vorgaben in Bezug auf die Darlehensvergabe zum Bau und Erwerb von Wohnimmobilien, die die Entstehung systemischer Risiken im Hypothekarkreditsystem verhindern und mögliche Verluste im Vorfeld einschränken sollen. In Abgrenzung zur mikroprudenziellen, einzelinstitutsspezifischen Regulierung, betreffen makroprudenzielle Instrumente den Finanzsektor in seiner Gesamtheit.

Im Sommer 2015 hat der Ausschuss für Finanzstabilität der Bundesregierung empfohlen, präventiv auch für Deutschland makroprudenzielle Instrumente für den Wohnimmobilienbereich zu schaffen (Ausschuss für Finanzstabilität, 2015). ${ }^{1}$ Im Gegensatz zu anderen europäischen Ländern waren diese bislang nicht vorhanden. Im Frühjahr 2017 hat die Bundesregierung nach mehrmonatigen Beratungen mit dem Bundesfinanzministerium (BMF), der Bundesbank und der Bundesanstalt für Finanzdienstleistungsaufsicht (BaFin) sowie der Anhörung von Sachverständigen und Spitzenverbänden der Kredit- und Immobilienwirtschaft einen Gesetzentwurf für ein entsprechendes Finanzaufsichts-

\footnotetext{
${ }^{1}$ Die Empfehlung erfolgte auf der Grundlage von $\S 3$ Absatz 2 des Gesetzes zur Überwachung der Finanzstabilität vom 28. November 2012 (BGBI. I, S. 2369), geändert durch Artikel 21 des Gesetzes vom 4. Juli 2013 (BGBI. I, S. 1981) („Finanzstabilitätsgesetz (FinStabG“).
} 
rechtsänderungsgesetz beschlossen. ${ }^{2}$ Für den Fall, dass es infolge des dauerhaft niedrigen Zinsniveaus zu kreditfinanzierten Übertreibungen am deutschen Wohnimmobilienmarkt kommen sollte, sieht das Gesetz folgende Instrumente vor (Deutscher Bundestag, 2017):

- Die Vorgabe einer Obergrenze für das Verhältnis zwischen dem gesamten Fremdkapitalvolumen einer Immobilienfinanzierung und dem Marktwert der Immobilie zum Zeitpunkt der Darlehensvergabe (Kreditvolumen-Immobilienwert-Relation bzw. Loan-to-Value: LTV), sowie

- die Vorgabe eines Zeitraums, innerhalb dessen ein bestimmter Anteil des Darlehens spätestens getilgt werden muss, oder, im Fall von endfälligen Darlehen, einer maximalen Laufzeit (Amortisationsanforderung). ${ }^{3}$

Die BaFin wird ermächtigt, diese Vorgaben im Wege der Allgemeinverfügung jeweils einzeln oder in Kombination festzulegen. Sie kann zudem institutsspezifische Freikontingente sowie Bagatellgrenzen definieren. ${ }^{4}$ Von den Beschränkungen explizit ausgenommen sind Darlehen für den Aus- oder Umbau sowie für die Sanierung von Bestandsimmobilien, für Maßnahmen des sozialen Wohnungsbaus, Anschlussfinanzierungen sowie für die Umschuldung und Restrukturierung von notleidenden Darlehen (Deutscher Bundestag, 2017)..$^{5}$ Zwar betrifft der Geltungsbereich der Vorgaben grundsätzlich auch die gewerbliche Wohnimmobilienfinanzierung. Es jedoch ist davon auszugehen, dass insbesondere Kredite an Privathaushalte von den Regelungen betroffen sein werden, da es bei vielen institutionellen Anlegern, wie etwa Fonds, bereits Eigenkapitalvorgaben gibt.

Die Bundesregierung hat kommuniziert, dass aus ihrer Sicht derzeit keine akute Gefährdung der Finanzstabilität durch Entwicklungen am deutschen Wohnimmobilienmarkt vorliege. Zum gleichen Ergebnis kam auch der letzte Finanzstabilitätsbericht der Bundesbank vom November 2016 (Deutsche Bundesbank, 2016). Die neu eingeführten Vorgaben werden deshalb nicht unmittelbar bindend. Gleichwohl ist denkbar, dass die neu geschaffenen Regelungen bereits in mittelbarer Zukunft zum Einsatz kommen. Allerdings ist in Deutschland bisher weder eine massive Ausweitung der Bautätig-

\footnotetext{
2 „Entwurf eines Gesetzes zur Ergänzung des Finanzdienstleistungsaufsichtsrechts im Bereich der Maßnahmen bei Gefahren für die Stabilität des Finanzsystems und zur Änderung der Umsetzung der Wohnimmobilienkreditrichtlinie“ (Deutscher Bundestag, 2017). Im Einzelnen werden Änderungen im Kreditwesengesetz (KWG), im Kapitalanlagegesetzbuch (KAGB) und im Versicherungsaufsichtsgesetz (VAG) vorgenommen.

${ }^{3}$ Vorige Gesetzentwürfe sahen zusätzlich die Möglichkeit der Vorgabe einer Obergrenze für den Schuldendienst im Verhältnis zum Einkommen des Schuldners („Debt-Service-To-Income“: DSTI) bzw. in Form einer Untergrenze für den Schuldendienstdeckungsgrad („Debt-Service-Coverage-Ratio“: DSCR) sowie eine Obergrenze für das Verhältnis zwischen Gesamtverschuldung und Einkommen („Debt-To-Income“: DTI) vor.

${ }^{4}$ Die Bagatellgrenze liegt bei mindestens 50.000 Euro, d. h. Kredite bis zu mindestens dieser Höhe wären von der Beschränkung nicht betroffen. Darüber hinaus wurden zwei Schwellenwerte bei einem Beleihungswert (nach Definition \$16 Pfandbriefgesetz) von 200.000 und 400.000 Euro eingeführt. Ist bei diesen Schwellenwerten eine Besicherung des Kredits in Höhe von 80 Prozent bzw. 60 Prozent des Beleihungswertes gegeben, wäre auch ein solcher Kredit nicht der Regulierung unterworfen. Beim oberen Schwellenwert entspricht die Besicherungsanforderung der Beleihungsgrenze gemäß § 14 Pfandbriefgesetz.

${ }^{5}$ Zur Vermeidung von Regulierungsarbitrage und Wettbewerbsverzerrungen werden alle gewerblichen Darlehensgeber, d. h. Banken, Versicherungen und Kapitalverwaltungsgesellschaften, vom neuen Gesetz erfasst.
} 
keit privater Haushalte noch der Kreditvergabe feststellbar, wie es typisch für eine systemrelevante Überhitzung wäre (Deschermeier et. al, 2016, Bendel und Voigtländer, 2017).

Trotz der beschlossenen Ausnahmeregelungen würden Darlehensnehmer und Kreditinstitute im Fall der Einführung der neuen makroprudenziellen Instrumente vor Herausforderungen gestellt. Das deutsche System der Wohnimmobilienfinanzierung ist im internationalen Vergleich bereits jetzt konservativ ausgestaltet und durch das Privathaftungsprinzip geprägt, was den Aufbau kreditfinanzierter Übertreibungen bisher effektiv verhindert hat (Zhu et al., 2017). ${ }^{6}$ Hinzu treten neue Regulierungen wie Basel III und die Wohnimmobilienkreditrichtlinie, deren Mittel- und Langfristwirkungen noch nicht absehbar sind. Aus ökonomischer Perspektive stellen sich deshalb Fragen nach der Treffsicherheit und praktischen Umsetzbarkeit sowie den möglichen unerwünschten Nebeneffekten der beschlossenen Instrumente. Zudem besteht das Problem einer treffsicheren Diagnose finanzstabilitätsgefährdender Übertreibungen am Hypothekenmarkt, welche letztlich die Grundlage für den Einsatz der Instrumente bilden müsste.

Vor diesem Hintergrund untersucht das vorliegende Papier die Notwendigkeit einer makroprudenziellen Regulierung des deutschen Hypothekenmarktes. Wir kommen zu dem Ergebnis, dass ein Einsatz der neu geschaffenen makroprudenziellen Instrumente das Hauspreiswachstum wahrscheinlich effektiv absenken würde, angesichts der bereits heute stabilitätsorientierten Ausrichtung der Wohnimmobilienfinanzierung auf absehbare Zeit aber nicht erforderlich ist. Wir argumentieren, dass Entscheidungen für oder gegen einen Einsatz der neu geschaffenen Vorgaben grundsätzlich nicht diskretionär, sondern regelgebunden auf der Grundlage belastbarer Einzelkreditdaten erfolgen sollten. Diese sollten insbesondere auch die Anzahl von Vollfinanzierungen und Kreditausfällen berücksichtigen. Dies würde deutlich besser als bisher ermöglichen, den heterogenen Finanzierungspraktiken an den regionalen Wohnungsmärkten Rechnung zu tragen.

Das Papier ist wie folgt gegliedert: Zunächst erörtern wir die Motivation für eine makroprudenzielle Regulierung des Hypothekenmarktes sowie die bisherigen internationalen Erfahrungen mit einer solchen. Daraufhin befassen wir uns mit den möglichen Wirkungen und Nebenwirkungen der in Deutschland neu beschlossenen Instrumente. Anhand eines von Jordà et al. (2017) kürzlich publizierter Zeitreihendatensatzes schätzen wir, ob und inwieweit bei einer makroprudenziell bedingten Drosselung der Kreditvergabe auch eine Absenkung des Hauspreiswachstums wahrscheinlich wäre. Auf Basis von Mikrodaten des European Household Finance and Consumption Survey (HFCS) untersuchen wir im Anschluss, welche Immobilienfinanzierungsgewohnheiten bei deutschen Haushalten vorherrschen, auch im Vergleich zum europäischen Ausland. Dies ermöglicht Aussagen darüber, wie

\footnotetext{
${ }^{6}$ Zhu et al., 2017 schätzen auf Basis der Taylor-Regel und Abweichungen von fundamental gerechtfertigten Hauspreisen, dass die Hauspreise in Spanien und Irland trotz sehr expansiver europäischer Geldpolitik um 16$35 \%$ niedriger wären, wenn die Hypothekenmärkte ähnlich konservativ wie in Deutschland ausgerichtet wären.
} 
viele und insbesondere welche Typen von Haushalten von den neuen Vorgaben betroffen wären. Auf Basis einer Betrachtung bestehender Regulierungen im deutschen Wohnimmobilienfinanzierungssystem stellen wir abschließend die Frage, ob der Nutzen der makroprudenziellen Instrumente bei einer Einführung ihre Kosten übersteigen würde, sodass sich eine Einführung ökonomisch rechtfertigen ließe.

\section{Konzept der makroprudenziellen Regulierung des Hypothekenmarktes}

\subsection{Motivation makroprudenzieller Instrumente}

Die Motivation für eine makroprudenzielle Regulierung des deutschen Hypothekenmarktes speist sich in erster Linie aus jüngeren Erfahrungen mit kreditfinanzierten Übertreibungen an nationalen Immobilienmärkten außerhalb Deutschlands. Diese Übertreibungen bauten sich typischerweise über längere Zeiträume auf. Kam es infolge veränderter makroökonomischer Rahmenbedingungen oder eines erkennbaren Überangebots zu einer plötzlichen Korrektur, konnte dies die Finanzstabilität massiv gefährden und in schweren, zum Teil weltweit spürbaren Finanzkrisen münden. Die entsprechenden Krisen, allen voran die vom amerikanischen Wohnimmobilienmarkt ausgehende Finanzkrise, wirkten äußerst destruktiv auf die volkswirtschaftliche Wohlfahrt (Jordà et al., 2015a; 2015b).

Eine wesentliche Ursache für die Entstehung kreditfinanzierter Übertreibungen an den Immobilienmärkten waren Lockerungen der Finanzierungsbedingungen, welche die Hypothekenkreditvergabe an Haushalte und die Immobilienpreise steigen ließen. So führten etwa in den USA eine Reihe von Leitzinssenkungen in Kombination mit einer Deregulierung der Hypotheken- und Refinanzierungsmärkte zu sinkenden Fremdkapitalkosten und einer Absenkung von Kreditvergabestandards. Zudem machten die Möglichkeit des steuerlichen Schuldzinsenabzugs sowie neuartige Kreditprodukte Immobilienfinanzierungen mit hohen Fremdkapitalanteilen attraktiv (Allen, 2005; Jordà et al., 2015b; Cerutti et al., 2017a). Die dadurch induzierte zusätzliche Immobiliennachfrage ging vor allem in Großstädten mit hohen Preissteigerungen einher, da das Immobilienangebot durch Baulandknappheit und zeitintensive Genehmigungsprozesse verspätet reagierte. Dem Preissignal folgend, investierten rasch weitere Haushalte in Wohnimmobilien. ${ }^{7}$ Dies wiederum löste einen Bauboom aus, welcher Arbeitskräfte band und die gesamtwirtschaftliche Produktionsstruktur zugunsten des Bau-, Kredit- und Immobiliensektors veränderte. Infolge der positiven Einkommensentwicklung und der Erwartung stetig steigender Immobilienwerte kam ein positiver Rückkopplungsprozess in Gang. Dieser Prozess wurde

\footnotetext{
${ }^{7}$ Empirische und experimentelle Forschung zeigen, dass private Investoren an Wohnimmobilienmärkten in der Regel adaptive Erwartungen bilden (Case et al., 2012; Armona et al., 2016). Dies trägt zur hohen zeitlichen Persistenz („Momentum“) von Immobilienpreiszuwächsen bei.
} 
letztlich durch ein steigendes Zinsumfeld gestoppt, infolge dessen viele Kreditnehmer ihre oft mit variablem Zinssatz versehenen Kredite nicht mehr bedienen konnten.

Seit der Nachkriegszeit haben Häufigkeit und Amplituden vergleichbarer Boom-Bust-Zyklen weltweit zugenommen. In vielen Ländern sind die Hypothekarschulden relativ zum Wert des Wohnungsbestands und der gesamtwirtschaftlichen Produktion langfristig angestiegen („Great Mortgaging“, Jordà et al., 2016). Ursache war einerseits eine oft ausufernde Wohneigentumsförderung, welche die Attraktivität der Fremdfinanzierung von Wohnimmobilien etwa durch die Möglichkeit des Schuldzinsenabzugs erhöhte. Auf der Bankenseite haben Regulierungsmaßnahmen wie Basel I/II darüber hinaus dazu beigetragen, das Wohnimmobilienfinanzierungsgeschäft als vergleichsweise risikolos anzusehen, da verhältnismäßig wenig Eigenkapital für entsprechende Kredite zu hinterlegen ist. ${ }^{8}$ Insgesamt hat dies zu einer stetig wachsenden Relevanz des Wohnimmobilienmarktes und des Hypothekenkreditsystems für die Finanzstabilität geführt (Ausschuss für Finanzstabilität, 2015).

In Deutschland hat es seit der Nachkriegszeit keine das Finanzsystem gefährdende Krise am Wohnimmobilienmarkt gegeben. Auch aufgrund einer niedrigen Wohneigentumsquote ist die Gesamtverschuldung der privaten Haushalte mit Hypothekenkrediten, bezogen auf das verfügbare Einkommen, zurzeit deutlich geringer als in anderen europäischen Ländern oder den Vereinigten Staaten (European Mortgage Federation, 2016). Dennoch bilden Wohnimmobilienkredite volumenmäßig die wichtigste Kreditart im deutschen Bankensystem.

\subsection{Bisherige internationale Erfahrungen}

Erfahrungen mit einer makroprudenziellen Regulierung der Wohnimmobilienfinanzierung bestehen bislang vor allem in Transformations- und Schwellenländern. ${ }^{9}$ Die eingesetzten Instrumente reichen von Anforderungen an die Eigenkapitalunterlegung von Immobilienkrediten bei Finanzierungsgebern bis hin zu am einzelnen Kreditnehmer beziehungsweise der finanzierten Immobilie ansetzenden Instrumenten (vgl. Tabelle 1). In der jüngeren Vergangenheit haben auch mehrere Industrieländer, darunter Mitgliedsstaaten der Europäischen Union und der Eurozone, makroprudenzielle Instrumente eingeführt. Begrenzungen der Kredit-Markwert-Relation (LTV) werden besonders häufig genutzt (Cerutti et al. 2017b). Zum Teil existieren auch Beschränkungen der Kredithöhe-EinkommensRelation (Debt-To-Income, DTI) oder der Zins- und Tilgungsbelastung relativ zum Einkommen (DebtService-To-Income, DSTI (Hartmann, 2015). Klar erkennbar ist eine Bevorzugung von kreditnehmer-

\footnotetext{
${ }^{8}$ Zudem besteht im Rahmen von Basel III über die "Capital Requirements Regulation" die Möglichkeit, Immobiliarkredite bis zu einer Beleihungsgrenze von 80 Prozent, statt wie zuvor 60 Prozent, privilegiert zu behandeln.

${ }^{9}$ Gründe dafür sind eine oft vorherrschende Kopplung der heimischen an eine ausländische Währung, ein vergleichsweise geringer Entwicklungsgrad der heimischen Hypothekenmärkte und die häufige Verwendung von Fremdwährungskrediten (Cerutti et al., 2017b).
} 
gegenüber kapitalbasierten und alternativen Instrumenten. Letztere werden in Europa durch die Credit Requirements Directive (CRD IV) und die Capital Requirement Regulation (CRR) gesteuert. ${ }^{10}$

\begin{tabular}{|c|c|c|}
\hline Instrument(e) & Zielrichtung & Mögliche Nebeneffekte \\
\hline $\begin{array}{l}\text { Antizyklische Kapitalunterle- } \\
\text { gungsvorschriften für Hypothe- } \\
\text { kenkredite }\end{array}$ & $\begin{array}{l}\text { Steigerung der Fremdfinanzie- } \\
\text { rungskosten für Immobilien in } \\
\text { Boomphasen, gleichzeitig Bil- } \\
\text { dung von Kapitalpuffern für } \\
\text { Abschwungphasen }\end{array}$ & $\begin{array}{l}\text { Rationierung der Kredit- } \\
\text { vergabe durch Banken }\end{array}$ \\
\hline $\begin{array}{l}\text { Höhere (dynamische) Risikoge- } \\
\text { wichte für Hypothekenkredite }\end{array}$ & s. o. & s. o. \\
\hline $\begin{array}{l}\text { Direkte Beschränkung des Hypo- } \\
\text { thekenkreditwachstums }\end{array}$ & $\begin{array}{l}\text { Begrenzung des Verschuldungs- } \\
\text { grads der Darlehensnehmer, } \\
\text { Senkung des Hauspreiswachs- } \\
\text { tums }\end{array}$ & $\begin{array}{l}\text { Ausschluss von Haushalten } \\
\text { von der Wohneigentumsbil- } \\
\text { dung }\end{array}$ \\
\hline $\begin{array}{l}\text { Direkte Beschränkung der Spezia- } \\
\text { lisierung von Banken auf Immobi- } \\
\text { lienkredite }\end{array}$ & $\begin{array}{l}\text { Begrenzung der Verwundbar- } \\
\text { keit von Banken durch sektor- } \\
\text { spezifische Schocks }\end{array}$ & $\begin{array}{l}\text { Steigerung der Fremdfinan- } \\
\text { zierungskosten für Immobi- } \\
\text { lien aufgrund von Effizienz- } \\
\text { verlusten }\end{array}$ \\
\hline $\begin{array}{l}\text { Obergrenzen für LTV, DTI, DSTI } \\
\text { und verwandte Maßnahmen }\end{array}$ & $\begin{array}{l}\text { Begrenzung des Verschuldungs- } \\
\text { grads der Darlehensnehmer, } \\
\text { Senkung des Hauspreiswachs- } \\
\text { tums, Senkung der Ausfallwahr- } \\
\text { scheinlichkeit }\end{array}$ & $\begin{array}{l}\text { Ausschluss von Haushalten } \\
\text { von der Wohneigentumsbil- } \\
\text { dung }\end{array}$ \\
\hline
\end{tabular}

Tabelle 1: Makroprudenzielle Instrumente für den Hypothekensektor und ihre Wirkungen. Quelle: Eigene Darstellung in Anlehnung an Crowe et al. (2013)

Aufgrund relativ kurzer Feldphasen und der oftmals eingeschränkten Datenverfügbarkeit können die Wirkungen makroprudenzieller Instrumente wissenschaftlich erst seit Kurzem intensiv erforscht werden. Vorhandene Studien beruhen überwiegend auf makroökonomischen Daten. Sie unterscheiden sich im Hinblick auf drei Dimensionen: die analysierten Instrumente, die untersuchten Länder sowie die untersuchte(n) Zielgröße(n). Bei den Zielgrößen dominieren das gesamtwirtschaftliche Hypothekenkreditwachstum und das (typischerweise inflationsbereinigte) nationale Hauspreiswachstum.

Crowe et al. (2013) analysieren die Eignung makroprudenzieller Instrumente zur Eindämmung kreditfinanzierter Übertreibungen an Wohnimmobilienmärkten anhand einer Vielzahl länderbezogener Fallstudien. Ihre Ergebnisse sind insgesamt ambivalent: Während einige Instrumente in bestimmten Ländern durchaus effektiv waren, erzielten sie in anderen nicht die gewünschte Wirkung. Dabei spielte der geldpolitische Kurs während der Implementierungsphase eine Rolle: War dieser Kurs zu expansiv, verfehlten die makroprudenziellen Instrumente oftmals ihre intendierte Wirkung. Die Einführung einer LTV-Obergrenze oder verwandter kreditnehmerbasierter Instrumente (insbesondere DTI) stu-

\footnotetext{
${ }^{10}$ Beispiele sind der im Rahmen von Basel III beschlossene antizyklische Kapitalpuffer sowie vorgeschriebene Liquiditätsquoten und maximale Amortisationsanforderungen für Geschäftsbanken.
} 
fen die Autoren als vergleichsweise effektiv ein. Ein dämpfender Effekt auf Kreditvolumen und Hauspreiswachstum sei allerdings oftmals lediglich temporär vorhanden.

Cerutti et al. (2017b) erweitern die vorgenannte Studie auf Basis einer Umfrage des Internationalen Währungsfonds (IWF) unter nationalen Aufsichtsbehörden. ${ }^{11}$ Ihre Analyse deckt zwölf verschiedene Instrumente in 119 Entwicklungs-, Schwellen- und Industrieländern für den Zeitraum 2000-2013 ab, darunter auch Obergrenzen für den Fremdfinanzierungsanteil. Die Studie zeigt, dass alle Arten makroprudenzieller Instrumente in den untersuchten Ländern zunehmend häufiger implementiert werden. Die Nutzung solcher Instrumente ist während einer Aufschwungphase am Wohnimmobilienmarkt mit statistisch signifikant geringeren Kreditwachstumsraten assoziiert. Zwar gilt dies unabhängig von der Höhe des nationalen Pro-Kopf-Einkommens, doch ist die Stärke des Zusammenhangs für Industrieländer am geringsten. Eine Begrenzung des Fremdfinanzierungsanteils bei Neukrediten geht unabhängig vom Pro-Kopf-Einkommen mit einer signifikant geringeren Wachstumsrate der Kredite an Privathaushalte einher, nicht jedoch mit einem geringeren Wachstum der Hauspreise.

Zu ähnlichen Ergebnissen kommen Kuttner und Shim (2016). Ihre Studie untersucht die Wirkung makroprudenzieller Instrumente auf Basis des bislang in der Literatur umfangreichsten Datensatzes, eines Makropanels mit 57 Ländern und zum Teil bis in die 1980er Jahre zurückreichender Historie. Die Untersuchung unterscheidet sich von den vorgenannten dahingehend, dass sie neben makroprudenziellen Vorgaben auch fiskalische Instrumente in die Betrachtung einbezieht, insbesondere die Subventionierung von Wohneigentum und die Besteuerung von Immobilientransaktionen. Zur Absenkung der Neukreditvergabe eignet sich ihren Ergebnissen zufolge insbesondere die Einführung oder Verschärfung einer DSTI-Obergrenze. Da Obergrenzen für LTV und DSTI in den betrachteten Ländern allerdings häufig simultan eingeführt oder verändert wurden, können sie einen eigenständigen Effekt einer LTV-Obergrenze gleichwohl nicht ausschließen. Zur Begrenzung des Hauspreiswachstums erwiesen sich dagegen vor allem Grunderwerbssteuererhöhungen als effektiv.

Zwei der wenigen auf Mikrodaten basierenden Untersuchungen sind die Studien von Claessens et al. (2013) sowie Jácome und Mitra (2015). Claessens et al. (2013) untersuchen anhand eines internationalen Bankpanels (darunter auch Banken in 24 Industrieländern) die Wirkungen makroprudenzieller Instrumente auf die Bilanzen einzelner Banken und die Stabilität nationaler Bankensysteme. Darlehensnehmerbasierte Regulierungsmaßnahmen reduzieren ihrer Analyse zufolge effektiv das Wachstum der Gesamtverschuldung, der Assets und des Verhältnisses von Nichtkernschuldtiteln zu Kernschuldtiteln von Banken. Sie führen in der Konsequenz zu geringen systemischen Risiken im Verlauf von Boomphasen am Immobilienmarkt. In Rezessionen wirkte sich eine Aussetzung oder Abschwä-

\footnotetext{
11 „Survey on Global Macroprudential Policy Instruments“ (GMPI).
} 
chung jedoch nur bei wenigen Instrumenten mildernd aus. Dieses Ergebnis findet sich auch bei Kuttner und Shim (2016) sowie Cerutti et al. (2017b).

Jácome und Mitra (2015) fokussieren sich bei ihrer Analyse vollständig auf die Wirkung von Obergrenzen für LTV und DTI bei Wohnimmobilienkrediten. Auf Basis granularer Daten auf Einzelkreditebene aus sechs Schwellenländern können sie zeigen, dass beide Instrumente das Hypothekenkreditwachstum und die Kreditausfallwahrscheinlichkeiten in den meisten Fällen effektiv senken konnten. Die Wachstumsrate der Hauspreise wurde dagegen nur in wenigen Fällen beeinflusst.

Trotz methodischer Einschränkungen ${ }^{12}$ deutet die vorhandene Forschung darauf hin, dass die Einführung oder Verschärfung von Obergrenzen für den Beleihungsauslauf und verwandte kreditnehmerbasierter Instrumente (DTI, DSTI) die Wachstumsrate des Hypothekenkreditvolumens während einer Boomphase am Immobilienmarkt wirksam absenken kann. Für die Wachstumsrate der Hauspreise gilt dies offenbar nur eingeschränkt. Während eines Abschwungs kann durch die Aussetzung entsprechender Instrumente zudem keine Stabilisierung erreicht werden, die Instrumente wirken also asymmetrisch. Daraus ist zu schließen, dass die für Deutschland beschlossenen makroprudenziellen Instrumente bei einer Einführung in erster Linie die Ausbreitung systemischer Risiken während einer lang andauernden Aufschwungphase verringern könnten, also Vorsorgecharakter hätten.

\section{Makroprudenzielle Regulierung des deutschen Hypothekenmarktes?}

\subsection{Kreditwachstum und reale Häuserpreise in Deutschland}

Die vorhandene empirische Evidenz zur Wirksamkeit makroprudenzieller Instrumente lässt offen, ob und inwieweit die dort gefundenen Ergebnisse auf Deutschland übertragbar sind. Es erscheint grundsätzlich plausibel, dass das international häufig auftretende Ergebnis einer Absenkung des Kreditwachstums durch LTV-Begrenzungen und verwandte Instrumente auch für Deutschland Gültigkeit besäße. Doch welche Implikationen hätte dies für die Wohnimmobilienpreise? Um dies abzuschätzen, untersuchen wir im Folgenden, wie eng das reale Hauspreiswachstum in Deutschland historisch mit der realen Wachstumsrate der Hypothekenkreditmenge partiell assoziiert war. Dazu verwenden wir einen neuen makrofinanziellen Datensatz der Universität Bonn (Jordá et al., 2017 sowie Knoll et al., 2017). Der Datensatz umfasst Zeitreihen zahlreicher gesamtwirtschaftlicher Variablen von 18702013 für 17 Industrieländer, unter anderem Hauspreise und Hypothekenkredite an Haushalte.

Je enger der partielle Zusammenhang zwischen Kreditwachstum und Hauspreiswachstum (auch im Vergleich zu anderen Industrieländern), desto eher ist zu erwarten, dass im Zuge einer makropruden-

\footnotetext{
12 Insbesondere bei den makroökonomischen Untersuchungen ist Endogenität ein gewichtiges Problem.
} 
ziellen Begrenzung des Kreditwachstums auch mögliche Preisübertreibungen am deutschen Wohnimmobilienmarkt eingedämmt werden könnten. Geschätzt wird folgende Gleichung anhand der Methode der kleinsten Quadrate (OLS):

$\Delta h p r_{t}=\alpha_{0}+\beta_{1} \Delta p o p_{t}+\beta_{2} \Delta g d p p c r_{t}+\beta_{3} \Delta$ mortr $_{t}+\beta_{4} D^{70 \_18}+\beta_{5} D^{19 \_45}+\beta_{6} D^{46 \_90}+\varepsilon_{t}$

Dabei bezeichnen $\Delta h p r_{t}$ das um Änderungen der Verbraucherpreisniveaus bereinigte Wachstum eines nationalen Hauspreisindex, $\Delta p o p_{t}$ das Bevölkerungswachstum, $\Delta g d p p c r_{t}$ das preisbereinigte Wachstum des Bruttoinlandsprodukts pro Kopf und $\Delta$ mortr $_{t}$ das preisbereinigte Wachstum der Hypothekenkreditmenge, gemessen jeweils in Prozent im Vergleich zum Vorjahr. ${ }^{13} D^{70 \_18}, D^{19 \_45}$ und $D^{46-90}$ sind Dummy-Variablen, die für die Zeiträume 1870-1918 beziehungsweise 1919-1945 oder 1946-1990 jeweils den Wert eins und sonst den Wert null annehmen und der Modellierung von Strukturbrüchen im Hauspreiswachstum dienen. Der Zeitraum 1991-2013 dient als Basiszeitraum. Der Fehlerterm $\varepsilon_{t}$ folgt einer Normalverteilung mit Erwartungswert null und heteroskedastischer Varianz, $\varepsilon_{t} \sim\left(0, \sigma_{t}^{2}\right)$. Tabelle 2 zeigt die Ergebnisse der Schätzung:

\begin{tabular}{llcc}
\hline & Koeffizient & t-Statistik (robust) & 95\%-Konfidenzintervall \\
\hline$\Delta p o p_{t}$ & $2,375^{*}$ & 1,89 & $(-0,118,4,867)$ \\
$\Delta g d p p c r$ & $-0,252$ & $-0,95$ & $(-0,777,0,273)$ \\
$\Delta$ mortr $_{t}$ & $0,185^{* *}$ & 2,58 & $(0,042,0,327)$ \\
$D^{70 \_18}$ & $-0,030$ & $-1,04$ & $(-0,089,0,028)$ \\
$D^{19 \_45}$ & $-0,030$ & $-0,72$ & $(-0,112,0,053)$ \\
$D^{46-90}$ & 0,021 & 1,35 & $(-0,010,0,052)$ \\
Konstante & $-0,010$ & $-1,28$ & $(-0,026,0,006)$ \\
Anzahl Beobachtungen & 100 & & \\
$R^{2}$ & 0,176 & & \\
F-Test (6,93) & $2,81^{* *}$ & & \\
Durbin-Watson-Statistik & 1,87 & & \\
\hline
\end{tabular}

Tabelle 2: Output einer OLS-Schätzung von Gleichung (1) mit Daten für Deutschland von 18702013. Die abhängige Variable ist das prozentuale reale Hauspreiswachstum gegenüber dem Vorjahr. ${ }^{*}, * *, * * *$ kennzeichnen statistische Signifikanz auf dem 10-, 5- bzw. 1-Prozentniveau.

Quelle: Jordá et al. (2017), eigene Berechnungen.

Gemäß dem für $\beta_{3}$ geschätzten Wert geht in Deutschland eine Steigerung des realen Hypothekenkreditwachstums um einen Prozentpunkt im Durchschnitt mit einem knapp 0,2 Prozentpunkte höheren realen Hauspreiswachstum im selben Jahr einher. Auch unter Kontrolle weiterer Determinanten

\footnotetext{
${ }^{13}$ Da die Zeitreihen in ihren Absolutwerten jeweils Einheitswurzeln aufweisen, verwenden wir zur Schätzung ausschließlich Wachstumsraten. Diese sind Stationaritätstests zufolge als stationäre Zeitreihen anzusehen.
} 
des Hauspreiswachstums besteht somit empirische Evidenz für einen signifikanten und ökonomisch bedeutsamen Zusammenhang zwischen Hypothekenkreditmenge und Hauspreiswachstum. Wie ein Modell mit gepoolten Daten zeigt, war dieser Zusammenhang etwas weniger stark als in anderen Industrieländern. ${ }^{14}$

Deutschland unterscheidet sich allerdings deutlich von anderen Volkswirtschaften, wenn die Absolutwerte der Wachstumsraten beider Variablen betrachtet werden. Wie Tabelle 3 zeigt, weist Deutschland hier im internationalen Vergleich tendenziell unterdurchschnittliche Werte auf. Für das Wachstum der Hypothekenkredite gilt dies insbesondere für den Zeitraum 2000-2013, in dem die Medianwachstumsrate real negativ war. Auch das reale Wachstum der Hauspreise war nicht nur weit niedriger als in anderen Ländern, sondern ab 2000 im Durchschnitt ebenfalls negativ. ${ }^{15}$ Auf Basis eines Fehlerkorrektur-Modells zeigen Bendel und Voigtländer (2017) überdies, dass die gesamtwirtschaftliche Kreditvergabe unter Berücksichtigung der Entwicklung der Hauspreise und Zinsen in Deutschland zuletzt unterproportional war. Anders als etwa in Spanien, dem Vereinigten Königreich oder Irland hat die Kombination aus rückläufigen Langfristzinsen und steigenden realen Hauspreisen in Deutschland also bislang nicht zu einer übermäßigen Expansion der Kreditvergabe geführt.

\begin{tabular}{lllllll}
\hline & $\mathbf{1 8 7 0 - 2 0 1 3}$ & \multicolumn{3}{c}{$\mathbf{1 9 4 6 - 2 0 1 3}$} & $\mathbf{2 0 0 0 - 2 0 1 3}$ \\
\hline \multirow{2}{*}{ Deutschland } & $\emptyset(\Delta h p r)$ & $\emptyset(\Delta$ mort $)$ & $\emptyset(\Delta h p r)$ & $\emptyset(\Delta$ mort $)$ & $\emptyset(\Delta h p r)$ & $\emptyset(\Delta$ mort $)$ \\
\multirow{2}{*}{16 Industrieländer } & $0,77 \%$ & $6,15 \%$ & $2,87 \%$ & $5,69 \%$ & $-1,79 \%$ & $-1,66 \%$ \\
\hline & $1,63 \%$ & $6,21 \%$ & $2,26 \%$ & $8,18 \%$ & $2,51 \%$ & $4,60 \%$ \\
\hline
\end{tabular}

Tabelle 3: Medianwerte für das reale Hauspreiswachstum und das reale Hypothekenkreditwachstum in Deutschland und 16 Industrieländern, verschiedene Zeiträume.

Quelle: Jordá et al. (2017), eigene Berechnungen.

\subsection{Immobilienfinanzierungsgewohnheiten der privaten Haushalte}

Auch wenn eine makroprudenzielle Drosselung der Kreditvergabe vermutlich in der Lage wäre, das gesamtwirtschaftliche Hauspreiswachstum in Deutschland abzusenken, bedeutet dies noch keine Notwendigkeit für die Anwendung der neu geschaffenen Instrumente. Für die Überprüfung der Notwendigkeit ist nicht nur das Volumen der Kreditvergabe insgesamt zu betrachten, sondern auch die Art der Finanzierung auf der Mikroebene. Im Kern sollen makroprudenzielle Instrumente erstens verhindern, dass es zu einer kreditgetragenen Expansion der Immobilienpreise kommt, die zweitens bei einer Korrektur der Preise zu einer Überschuldung der Haushalte beitragen würde, die dann letztlich auf die Banken über notwendige Zwangsvollstreckungen zurückfiele. Entsprechend ist zu prüfen,

\footnotetext{
${ }^{14} \mathrm{Vgl}$. Tabelle A1 im Anhang.

${ }^{15}$ Auch demografische Einflussfaktoren und fiskalische Veränderungen trugen hierzu bei (Voigtländer, 2009). Ein weiterer Grund ist die ausgleichende Funktion eines wettbewerbsfähigen Mietmarktes (Lerbs, 2014).
} 
ob und inwiefern im deutschen Markt überhaupt Überschuldungstendenzen und risikobehaftete Kredite im Wohnungsmarkt vorliegen. Grundsätzlich werden Immobilienfinanzierungen vor allem durch die Art der Zinsbindung, die Tilgung sowie die Fremdkapitalquote charakterisiert.

Die Zinsbindung ist ein für die Finanzstabilität besonders wichtiger Faktor. Schließlich kam es in den USA, dem Vereinigten Königreich oder auch Spanien vor allem deswegen zu vermehrten Kreditausfällen, weil die im Vorfeld der Finanzkrise anziehenden Zinsen sich aufgrund überwiegend variabel verzinslicher Darlehen unmittelbar auf die Zinsbelastung der Haushalte auswirkten. Teilweise kam es zu einer Verdopplung der Zinsbelastung, auch weil zum Beispiel in den USA typischerweise Rabatte auf die Zinsbelastung in den ersten beiden Jahren gewährt wurden, die dann in Zeiten steigender Zinsen ausliefen (Jäger und Voigtländer, 2009). Feste Zinsbindungen hingegen gewähren den Darlehensnehmern Planungssicherheit und bei entsprechender Tilgung ist auch bei gestiegenen Zinsen zum Zeitpunkt der Anschlussfinanzierung eine Erhöhung der Zinsbelastung vermeidbar.

Mikrodaten auf Basis von Meldungen einzelner Banken sowie Surveys privater Haushalte zeigen, dass in Deutschland feste Zinsbindungen vorherrschend sind. Nach aktuellen Daten der Bundesbank ist der Anteil der Kredite mit einer Zinsbindungsfrist von über zehn Jahren von zwanzig Prozent aller neu vergebenen Hypothekenkredite im Jahr 2004 auf über 44 Prozent im Jahr 2016 gestiegen (Deutsche Bundesbank, 2017b). Besonders seit Anfang 2014 ist ein Trend zu längeren Zinsbindungen deutlich zu erkennen. Lediglich zehn Prozent aller neu ausgereichten Wohnungsbaukredite sind derzeit variabel verzinst oder haben eine Zinsbindung von bis zu einem Jahr. Daten des Household Finance and Consumption Survey (HFCS) der Europäischen Zentralbank belegen die Dominanz von Festzinsdarlehen auch bei Bestandskrediten: Laut aktuellstem Datenstand aus dem Jahr 2014 wurde bei 60 Prozent aller in diesem Jahr ausstehenden Hypothekenkredite eine Zinsbindung von zehn Jahren vereinbart, bei weiteren zwanzig Prozent waren es sogar mehr als zehn Jahre.

Zur Tilgung gibt es bislang keine detaillierten amtlichen Daten. Im Rahmen der HFCS werden auf der Haushaltsebene zwar Angaben zu aktuellen Zins- und Tilgungszahlungen sowie zur bisherigen Tilgungsleistung erhoben. Zins- und Tilgungszahlungen sind jedoch in einer aktuell gezahlten Rate zusammengefasst, so dass eine separate Auswertung der Tilgungsleistung - insbesondere der anfänglichen Tilgung - nicht verlässlich möglich ist. Daten der Plattform „Europace“, über die nach Angaben des Betreibers etwa 10 Prozent aller Wohnimmobilienkredite in Deutschland vermittelt werden, zeigen derweil in den letzten Jahren einen Anstieg der anfänglichen Tilgung auf ein hohes Niveau: im Mai 2017 lag die anfängliche Tilgung demnach im Durchschnitt bei knapp unter drei Prozent (Europace, 2017). Der Anteil tilgungsfreier Darlehen ist mit 13 Prozent zudem gering und vor allem konstant, im Gegensatz zu der Situation wie sie im Vorfeld der Finanzkrise etwa im Vereinigten Königreich zu beobachten war. 
Repräsentative Angaben zur Fremdkapitalquote zum Zeitpunkt des Immobilienerwerbs können auf Basis des HFCS berechnet werden. Demnach liegt die durchschnittliche Fremdkapitalquote aller Wohneigentümer mit Hypothekendarlehen nach dem letzten Datenstand aus dem Jahr 2014 in Deutschland mit 73 Prozent deutlich unter dem Niveau von etwa den Niederlanden (89 Prozent) oder Frankreich (78 Prozent). Gleichzeitig deuten die Daten darauf hin, dass zahlreiche Haushalte, die in Deutschland selbstgenutztes Wohneigentum erwerben, den Erwerb vollständig mit Fremdkapital finanzieren. Die Zahl der Haushalte mit Beleihungsausläufen von über 90 Prozent oder sogar über 100 Prozent zum Zeitpunkt des Immobilienerwerbs, die seit dem Jahr 2000 ein Hypothekendarlehen aufgenommen haben, ist im HFCS-Datensatz mit 20 bzw. 17 Prozent an allen Eigentümerhaushalten mit Hypothek überraschend hoch. ${ }^{16}$ Derart hohe Beleihungsquoten werden einerseits von Haushalten gewählt, die über hohe Vermögen verfügen (Bendel et al., 2016). Hohe LTVs finden sich zudem oftmals für relativ junge Haushalte, die Immobilien von vergleichsweise niedrigen Marktwerten erwerben: Für Hauskäufe ab dem Jahr 2000 liegt der durchschnittliche Kaufpreis bei Haushalten mit einer LTV zwischen 50 und 90 Prozent bei 196.000 Euro, wohingegen er bei Haushalten mit einer LTV von über 90 Prozent bei 135.000 Euro liegt. ${ }^{17}$

Des Weiteren zeigt sich, dass Haushalte mit höheren Beleihungsausläufen eher geringe Belastungen aus Zins und Tilgungen relativ zu ihrem Einkommen (DSTI) aufweisen. Wiederum für alle Wohnimmobilienkäufe ab 2000 lässt sich auf Basis des HFCS prüfen, wie hoch der Beleihungsauslauf zum Zeitpunkt des Kaufes war und wie groß gegenwärtig der Anteil der Zins- und Tilgungszahlungen am Einkommen ist. ${ }^{18}$ Weniger als die Hälfte der Haushalte mit einer LTV von über 90 Prozent weist demnach eine DSTI von mehr als 30 Prozent auf. Nur rund acht Prozent aller Wohneigentümer mit Hypothek haben gleichzeitig einen ursprünglichen Fremdfinanzierungsanteil von mindestens 90 Prozent und eine aktuelle DSTI von mehr als 30 Prozent. Abbildung 1 zeigt, dass der entsprechende Anteil in Frankreich, den Niederlanden oder Belgien jeweils deutlich über 15 Prozent liegt. In Österreich oder Italien ist diese Quote dagegen niedriger als in Deutschland. Allerdings muss berücksichtigt werden, dass gerade aufgrund der in Deutschland dominierenden Festzinsbindungen nur ein Teil der Haushalte tatsächlich höhere Lasten zu tragen hätte, wenn die Zinsen für Hypothekarkredite steigen würden. Haushalte mit hohen LTVs, aber geringen Belastungen im Verhältnis zu ihren Einkommen weisen gerade bei langen Zinsbindungen nur geringe Ausfallwahrscheinlichkeiten auf. Somit stellen sie allenfalls eine geringe Gefahr für die Finanzstabilität dar.

\footnotetext{
${ }^{16}$ Bei der Interpretation dieser Anteilswerte ist zu berücksichtigen, dass sie sich nur auf diejenigen Eigentümerhaushalte beziehen, die zur Finanzierung des Immobilienkaufs eine Hypothek aufgenommen haben. Ein hoher Anteil der Haushalte im HFCS-Datensatz gibt an, den Kauf vollständig aus Eigenmitteln finanziert zu haben.

${ }^{17}$ Die entsprechenden Medianwerte betragen 170.000 bzw. 120.000 Euro.

${ }^{18}$ Für die DSTI stehen lediglich Angaben zum Befragungszeitpunkt zur Verfügung, nicht zum Beginn der Finanzierung.
} 
Umgekehrt lassen die Mikrodaten des HFCS aber auch den Schluss zu, dass von einer Anwendung insbesondere des ersten neu geschaffenen makroprudenziellen Instruments - einer Begrenzung der LTV etwa auf 100 oder sogar 90 Prozent - ein vergleichsweise großer Anteil Eigentum erwerbender Haushalte betroffen wäre. Vor allem wären Haushalte betroffen, bei denen das Kreditrisiko aus Sicht der finanzierenden Banken in der Vergangenheit offenbar als gering eingestuft wurde und die Immobilien mit vergleichsweise geringen Marktwerten erwerben.

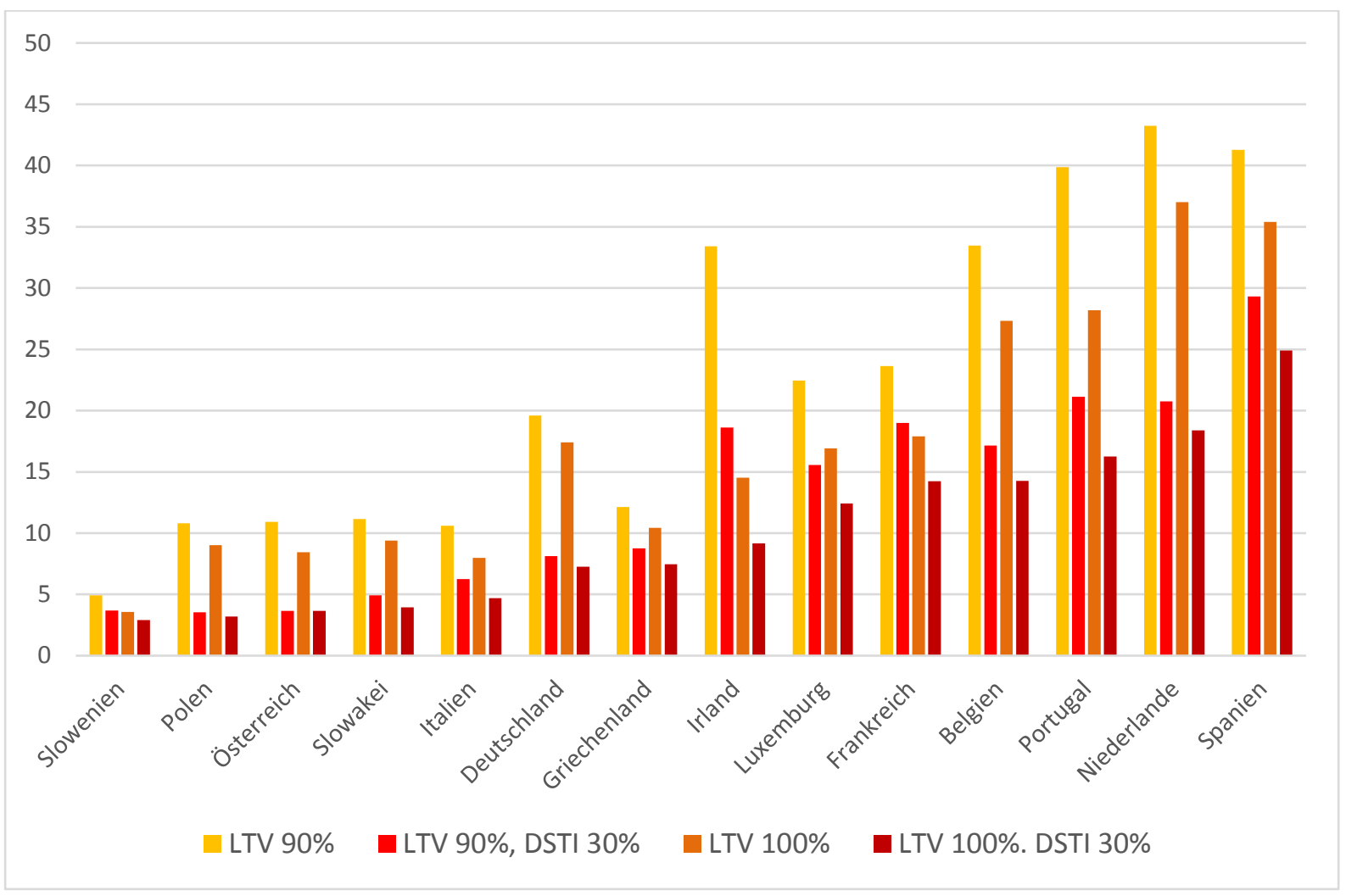

Abbildung 1: Anteil der deutschen Wohneigentümer mit Hypothekenfinanzierungen ab dem Jahr 2000, die jeweilige Schwellenwerte für LTV und DSTI überschreiten.

Quelle: HFCS, eigene Berechnungen

\subsection{Droht eine Überregulierung des deutschen Hypothekenmarktes?}

Vorhandene Daten zeigen, dass der Erwerb von Wohneigentum in Deutschland typischerweise mit langen Zinsbindungen, kontinuierlicher Tilgung und hohen Eigenkapitalanteilen finanziert wird. Gefahren einer Überschuldung der Haushalte im Falle sinkender Wohnungspreise sind also deutlich geringer als in anderen Ländern, obwohl ein nicht geringer Teil aller Haushalte, die für den Erwerb ein Darlehen aufnehmen, mit LTVs von 90 Prozent und mehr finanzieren. Diese Haushalte weisen typischerweise aber geringe Belastungen aus Zins- und Tilgung im Verhältnis zum Einkommen auf. Ein Grund dafür ist, dass sie tendenziell Objekte mit eher geringeren Marktwerten erwerben. Gerade für diese Haushalte sowie deren Kreditgeber sind erhebliche Konsequenzen bei einer Anwendung der 
makroprudenziellen Instrumente zu erwarten, die ins Verhältnis zu den Nutzen der Instrumente gesetzt werden müssen.

Zunächst einmal stellt die Einführung von rechtlichen Vorgaben bei der Eigenkapitalquote und der Amortisation einen starken Eingriff in die Vertragsfreiheit dar. Je nach den letztlich festgelegten Grenzwerten würde für einen erheblichen Teil der Haushalte der Zugang zu Krediten deutlich erschwert, ohne dass dies notwendigerweise die Finanzstabilität verbessern würde. Schließlich sind es oftmals Haushalte mit höheren Einkommen oder Vermögen, die hohe Beleihungsausläufe wählen, weil sie etwa noch keine Zeit zum Sparen oder ihr Vermögen in eher illiquide Anlagen investiert haben. Die zusätzlichen Restriktionen könnten sich damit negativ auf die Wohneigentumsbildung in Deutschland auswirken. Daten des sozioökonomischen Panels zeigen, dass die Wohneigentumsquote der jungen Haushalte zwischen 25 und 34 Jahren bereits von 18 Prozent im Jahr 2005 auf 13 Prozent im Jahr 2015 gesunken ist (Hude und Voigtländer, 2017). Weitere Hürden bei der Immobilienfinanzierung könnten die Vermögensbildung durch Wohneigentum zusätzlich erschweren, was unter anderem auch negative Rückwirkungen auf die Altersvorsorge hätte: Empirische Evidenz auf Grundlage der HFCS-Daten deutet darauf hin, dass die Sparquote deutscher Wohneigentümerhaushalte signifikant über derjenigen vergleichbarer Mieterhaushalte liegt (Schmidt und Le Blanc, 2017). Ein Grund hierfür ist, dass viele Wohneigentümer die Geldvermögensbildung nicht durch Kredittilgung substituieren, sondern trotz der Rückzahlung von Hypothekenkrediten zusätzlich sparen.

Nicht zuletzt würde die Beschränkung möglicher Beleihungsausläufe die infolge des Niedrigzinsumfelds unter Druck stehende Profitabilität der Banken weiter einschränken. Darlehen mit höheren Beleihungsausläufen sind typischerweise mit Zinsaufschlägen verbunden, die die Margen der Banken verbessern. Höhere Margen sind für die Banken essentiell, um Gewinne zu erzielen und damit die Eigenkapitalbasis zu erhöhen, welche wiederum grundlegend für eine höhere Robustheit ist. Entsprechend würde sich die Anwendung der Instrumente negativ auf die notwendige Stärkung der Eigenkapitalbasis auswirken. Dabei ist auch zu berücksichtigen, dass die Immobilienfinanzierung in Deutschland, wie auch in vielen anderen Ländern, bankenseitig aktuell auf eine neue regulatorische Basis gestellt wird: Basel III wird Schritt für Schritt implementiert und ist mit höheren Eigenkapitalanforderungen und strikteren Liquiditätsanforderungen verbunden (Hüther et al., 2015). Zeitgleich mit der Schaffung der makroprudenziellen Instrumente wurde außerdem die Wohnimmobilienkreditrichtlinie umgesetzt, die den Verbraucherschutz bei Immobiliendarlehen stärken und die Banken zu einer verantwortungsvolleren Kreditvergabe anhalten soll (Deutscher Bundestag, 2017). Beide Regelwerke setzen bereits Anreize zu einer vorsichtigeren Kreditvergabe. Auf Basis der Wohnimmobilienkreditrichtlinie ist es zudem möglich, dass Haushalte in Zahlungsschwierigkeiten zukünftig häufiger auf Kreditrückabwicklung klagen werden, wenn es Anhaltspunkte für eine zu optimistische Be- 
wertung der Einkommenssituation der Kreditnehmer zum Zeitpunkt des Darlehensabschlusses gibt. Entsprechend setzen diese Regulierungen schon starke Anreize, die Bonität von Kreditnehmern stärker zu prüfen und erschweren damit den Zugang zu Krediten (Dorffmeister, 2017). Die genauen Auswirkungen dieser Regeln gilt es zunächst abzuwarten, ehe eine Anwendung der makroprudenziellen Instrumente erwogen wird. Das Zusammenwirken aller in der jüngeren Vergangenheit auf Bank- und Darlehensnehmerseite geschaffenen Vorgaben kann letztlich nicht verlässlich abgeschätzt werden. Das Risiko negativer Auswirkungen auf Banken und Darlehensnehmer erscheint jedoch groß.

Zuletzt muss auch berücksichtigt werden, dass die potenzielle Anwendung der makroprudenziellen Instrumente bereits heute Unsicherheiten bei Banken auslöst. Noch ist weder bekannt, an welchen Kriterien oder Indikatoren eine Anwendung konkret festgemacht würde, noch auf welche Art und in welcher Höhe Grenzwerte und Kontingente bestimmt würden. Wie in Kapitel 3.2 gezeigt, würde es bereits einen großen Unterschied bedeuten, ob etwa nur 100-Prozent-Finanzierungen reglementiert würden oder auch schon Kredite mit Beleihungen von mehr als 90 Prozent. Die mangelnde Klärung dieser Detailfragen dürfte sich spätestens dann negativ auf die Kreditvergabe auswirken, wenn die Einführung der geschaffenen Instrumente unmittelbar bevorstünde.

Rational vergebene Hypothekenkredite stellen eine notwendige Bedingung für private Wohnungsbauinvestitionen dar, die wiederum ein nachhaltiges Wirtschaftswachstum sowie die Vermögensbildung der Haushalte befördern. Es ist somit insgesamt wahrscheinlich, dass die gesellschaftlichen Kosten den Nutzen der Regulierungen überschreiten würden.

\section{Schlussfolgerungen}

Die bisherigen internationalen Erfahrungen mit makroprudenziellen Instrumenten in der Immobilienfinanzierung sind insgesamt ambivalent. Insbesondere Begrenzungen des Beleihungsauslaufs können jedoch als ein effektives Instrument zur Vermeidung einer zu expansiven Kreditvergabe bei Immobilienbooms gelten. Die Einführung der Möglichkeit einer Begrenzung des Beleihungsauslaufs und der Vorgabe von Mindesttilgungen erscheint angesichts dieser Evidenz als passendes Instrument zur Vermeidung eines möglichen kreditbasierten Immobilienpreisbooms. Allerdings ist die Wohnimmobilienkreditvergabe in Deutschland bereits ohne diese Instrumente vorsichtig. Hohe Beleihungsausläufe werden mit Bedacht gewählt und kommen eher bei Haushalten mit höheren Vermögen und Immobilien mit vergleichsweise niedrigen Marktwerten zum Einsatz. Hinzu kommt, dass lange Zinsbindungen vorherrschend sind. Dadurch wird die Gefahr vermehrter Kreditausfälle infolge unerwarteter Zinsschocks moderiert. Letztlich wurden zudem in der jüngeren Vergangenheit bereits verschiedene Regulierungen eingeführt, deren Wirkungen auf die Kreditvergabe noch nicht evaluiert werden konn- 
ten. Vor diesem Hintergrund bestünde bei einer praktischen Einführung der neu geschaffenen makroprudenziellen Instrumente die Gefahr einer Überregulierung des deutschen Wohnimmobilienfinanzierungsmarktes. Diese hätte eine eingeschränkte Kreditvergabe zur Folge, durch die zwar das Hauspreiswachstum verlangsamt, insbesondere aber die Wohneigentumsbildung bei jüngeren Haushalten limitiert würde. Schon jetzt ist die Wohneigentumsbildung jüngerer Haushalte im internationalen Vergleich überaus niedrig und weiter rückläufig, was in Anbetracht des nach wie vor hohen Bedarfs an privater Altersvorsorge besorgniserregend ist.

Neben der Gefahr der Überregulierung stellt sich die Frage, nach welchen Kriterien eine Anwendung der makroprudenziellen Instrumente überhaupt erfolgen soll. Einen Einsatz an Entwicklungen im Immobilienmarkt zu koppeln erscheint kaum sinnvoll, da Verwerfungen im Kreditmarkt begrenzt werden sollen. Tatsächlich fehlt es der Finanzaufsicht aber hier an entsprechenden Daten. So gibt es derzeit weder amtliche noch sonstige repräsentative Daten zu Kreditausfällen sowie Tilgungen, Beleihungsausläufen oder Zinsbelastungen, die kurzfristig genug verfügbar wären. Mikrodaten aus Vermögensbefragungen können zwar genutzt werden, um durchschnittliche Niveaus und charakteristische Quantile zu ermitteln. Aufgrund der langen Zeitverzögerung zwischen Befragungszeitraum, Aufbereitung und Veröffentlichung sind diese Daten jedoch ungeeignet, aktuelle Entwicklungen abzubilden. Außerdem sind die Stichprobengrößen zu gering, um etwa Risiken in einzelnen regionalen Märkten erkennen zu können. Daher sollte vor einer tatsächlichen Anwendung der geschaffenen makroprudenziellen Instrumente zunächst das Monitoring des Immobilienfinanzierungsmarktes verbessert werden, um eine Entscheidung für oder wider einen Einsatz regelgebunden auf Basis verlässlicher Indikatoren zu ermöglichen. Dabei sind natürlich auch Kosten des Monitorings zu berücksichtigen. Anders als etwa Daten zum Einkommen können gerade Informationen zu Beleihungsausläufen, zur jeweiligen Zinsbindung, zur Anfangstilgung sowie zu Kreditausfällen vergleichsweise einfach bei den finanzierenden Banken gesammelt und der Aufsicht zur Verfügung gestellt werden. Anonymisiert können diese Daten dann auch für wissenschaftliche Zwecke genutzt werden. Dies würde es der Aufsicht auch erlauben, Veränderungen in der Kreditvergabepraxis einzelner Banken zu erkennen und auf dieser Basis das Gespräch mit der jeweiligen Bank zu suchen. Die Initiative Analytical Credit Datasets (AnaCredit) der Europäischen Zentralbank ist hier ein Anfang, sieht in ihrer ersten Stufe aber nur die Meldung von Krediten an Unternehmen vor. Ob und wann auch Immobiliendarlehen an private Haushalte erfasst werden sollen, ist derzeit offen. 


\section{Literatur}

Allen, F. (2005): Modelling Financial Instability, National Institute Economic Review, 192, S. 57-67.

Ausschuss für Finanzstabilität (2015): Empfehlung vom 30. Juni 2015 zu neuen Instrumenten für die Regulierung der Darlehensvergabe zum Bau oder Erwerb von Wohnimmobilien, Berlin.

Bendel, D., Demary, M. und M. Voigtländer (2016): Eine erste Bewertung makroprudenzieller Instrumente in der Immobilienfinanzierung, IW policy paper Nr. 8/2016, Köln.

Bendel, D. und M. Voigtländer (2017): Eine Risikoprüfung für die deutsche Wohnimmobilienfinanzierung, IW-Trends 43, S. 41-58.

Calomiris, C. W. (2008): The Subprime Turmoil: What's Old, What's new and What's Next. American Enterprise Institute for Public Policy Research, Washington.

Case, K. E., Shiller, R. J. und A. K. Thompson (2012): What Have They Been Thinking? Homebuyer Behavior in Hot and Cold Markets, Brookings Papers on Economic Activity, 2/2012, S. 265-315.

Cerutti, E., Dagher, J., Dell'Ariccia, G. (2017a): Housing Finance and Real-Estate Booms: A CrossCountry Perspective, Journal of Housing Economics 38, S. 1-13.

Cerutti, E., S. Claessens und L. Laeven (2017b): The use and effectiveness of macroprudential policies: New evidence, Journal of Financial Stability 28, S. 203-224.

Claessens, S., S. R. Ghosh und R. Mihet (2013); Macro-prudential policies to mitigate financial system vulnerabilities, Journal of International Money and Finance 39, S. 153-185.

Crowe, C. W., G. Dell'Ariccia, D. Igan und P. Rabal (2013): How to Deal with Real Estate Booms: Lessons from Country Experiences, Journal of Financial Stability 9, S. 300-319.

Deschermeier, P., Henger, R. Seipelt, B. und M. Voigtländer (2016):Zuwanderung, Wohnungsnachfrage und Baubedarfe - aktualisierte Ergebnisse des IW Wohnungsbedarfsmodells, IW-Report Nr. 16/2016, Köln

Deutsche Bundesbank (2016): Finanzstabilitätsbericht 2016, Frankfurt am Main.

Deutsche Bundesbank (2017a): Die Preise für Wohnimmobilien in Deutschland im Jahr 2016, Monatsbericht Februar 2017, S. 55-57.

Deutsche Bundesbank (2017b): Zinsbindungsfristen für Wohnungsbaukredite an private Haushalte in Deutschland, _https://www.bundesbank.de/Redaktion/DE/Downloads/Statistiken/Unternehmen_ Und_Private_Haushalte/Indikatorensystem_Wohnimmobilienmarkt/zinsbindungsfristen_fuer_wohn ungsbaukredite.png?_blob=publicationFile, letztmalig abgerufen am 21.6.2017.

Deutscher Bundestag (2017): Entwurf eines Gesetzes zur Ergänzung des Finanzdienstleistungsaufsichtsrechts im Bereich der Maßnahmen bei Gefahren für die Stabilität des Finanzsystems und zur Änderung der Umsetzung der Wohnimmobilienkreditrichtlinie (Finanzaufsichtsrechtergänzungsgesetz), Drucksache 18/10935, Berlin.

Dorffmeister, L. (2017): Die europäische Wohnimmobilienkreditrichtlinie, ifo Schnelldienst 1/2017, S. 41-44.

Europace (2017): EUROPACE Trendindikator Baufinanzierung, https://report.europace.de/ebixetb/europace-trendindikator-baufinananzierung-etb/, letztmalig abgerufen am 21.6.2017. 
European Mortgage Federation (2016): EMF Hypostat 2016: A Review of Europe's Mortgage and Housing Markets, Brüssel.

Gelain, P., K. J. Lansing und C. Mendicino (2013): House Prices, Credit Growth, and Excess Volatility: Implications for Monetary and Macroprudential Policy, International Journal of Central Banking 9 (2), S. 219-276.

Hartmann, P. (2015): Real Estate Markets and Macroprudential Policy in Europe, Journal of Money, Credit and Banking 47 (1), S. 69-80.

Hiller, N. (2014): Zinspolitik ade! Wie man Immobilienpreisblasen dennoch überstehen kann, Wirtschaftsdienst 10/2014, S. 748-755.

Hude, M. und M. Voigtländer (2017): Trends in der Wohneigentumsbildung, Gutachten des Instituts der deutschen Wirtschaft, Köln.

Hüther, M., Deschermeier, P., Haas, H. und M. Voigtländer (2015), Die Bedeutung der Langfristfinanzierung durch Banken, IW Analyse Nr. 101, Köln.

Jácome, Luis I. und S. Mitra (2015): LTV and DTI Limits - Going Granular, IMF Working Paper 15/154, Washington.

Jäger, M. und M. Voigtländer (2008): Hintergründe und Lehren aus der Subprime-Krise, IW-Trends 35, S. 17-29.

Jordà, Ò., Schularick, M. und A. M. Taylor (2015a): Betting the house, Journal of International Economics 96, S. 2-18.

Jordà, Ò., Schularick, M. und A. M. Taylor (2015b): Leveraged bubbles, Journal of Monetary Economics 76, S. 1-20.

Jordà Ò., Schularick M. und A. M. Taylor (2016): The Great Mortgaging: Housing Finance, Crises, and Business Cycles, Economic Policy 31 (85). S. 107-152.

Jordà, Ò., Schularick, M. und A. M. Taylor (2017): Macrofinancial History and the New Business Cycle Facts, in: Eichenbaum, M. und J. A. Parker (Hrsg.): NBER Macroeconomics Annual 2016, Chicago.

Knoll, K., Schularick, M. und T. Steger (2017): No price like home: global house prices, 1870-2012, American Economic Review 107 (2), S. 331-353.

Kuttner, K. N. und I. Shim (2012): Taming the real estate beast: the effects of monetary and macroprudential policies on housing markets and credit, in: Heath, A., F. Packer und C. Windsor (Hrsg.): Property Markets and Financial Stability, Sydney, S. 231-259.

Lerbs, O. (2014): Germany's rental property sector: A cornerstone of housing market resilience, Housing Finance International 2/2014, S. 38-44.

Reinhart, C. und K. Rogoff (2009): This Time Is Different: Eight Centuries of Financial Folly, Princeton.

Schmidt, T. und J. Le Blanc (2017): Do homeowners save more? Evidence from the Panel on Household Finances (PHF), Vortrag auf der European Real Estate Society (ERES) Conference 2017, Delft.

Voigtländer, M. (2009): Why is the German homeownership rate so low?, Housing Studies 24 (3), S. 355-372.

Zhu, B., Betzinger, M. und S. Sebastian (2017): Housing market stability, mortgage market structure, and monetary policy: Evidence from the euro area, Journal of Housing Economics 37, S. 1-21. 


\section{Anhang}

Tabelle A1: Ergebnis einer Pooled-OLS-Schätzung von Gleichung (1) mit den Paneldaten aller 16 Industrieländer im Datensatz ohne Deutschland: ${ }^{19}$

\begin{tabular}{|c|c|c|c|}
\hline & Koeffizient & t-Statistik (robust) & 95\%-Konfidenzintervall \\
\hline$\Delta p o p_{t}$ & $0,764^{*}$ & 1,81 & $(-0,063,1,591)$ \\
\hline$\Delta g d p p c r$ & $-0,022$ & $-0,38$ & $(-0,136,0,092)$ \\
\hline$\Delta m o r t r_{t}$ & $0,313 * * *$ & 6,97 & $(0,225,0,401)$ \\
\hline$D^{70 \_18}$ & $-0,023 * * *$ & $-3,25$ & $(-0,036,-0,009)$ \\
\hline$D^{19 \_45}$ & $-0,011$ & $-1,64$ & $(-0,024,0,002)$ \\
\hline$D^{46-90}$ & $-0,011^{*}$ & $-1,74$ & $(-0,023,0,001)$ \\
\hline Konstante & $-0,008$ & $-0,81$ & $(-0,026,0,011)$ \\
\hline Länder-Fixed-Effects & Ja & & \\
\hline Anzahl Beobachtungen & 1.586 & & \\
\hline$R^{2}$ & 0,101 & & \\
\hline F-Test $(21,1564)$ & $5,62 * * *$ & & \\
\hline
\end{tabular}

Tabelle A1: Output einer Pooled-OLS-Schätzung von Gleichung (1), erweitert um individuelle Achsenabschnitte, mit Paneldaten für 16 Industrieländer (ohne Deutschland) von 1870-2013. Die abhängige Variable ist das prozentuale reale Hauspreiswachstum gegenüber dem Vorjahr. ${ }^{*}, * *, * *$ kennzeichnen statistische Signifikanz auf dem 10-, 5- bzw. 1-Prozentniveau.

Quelle: Jordá et al. (2017), eigene Berechnungen.

19 Für die entsprechende Schätzung wurde das in Gleichung (1) beschriebene Schätzmodell um DummyVariablen für 15 Länder erweitert, um unbeobachtete Unterschiede in der Höhe des langfristigen Hauspreiswachstums zu berücksichtigen. Die geschätzten Koeffizienten für diese Dummy-Variablen werden in der Tabelle aus Platzgründen nicht ausgewiesen, können von den Autoren aber auf Anfrage zur Verfügung gestellt werden. 